\title{
Consistency in macroscopic human brain responses to noisy time-varying visual inputs
}

\author{
Authors: Keiichi Kitajo ${ }^{1,2,3 *}$, Takumi Sase ${ }^{1}$, Yoko Mizuno ${ }^{1}$, Hiromichi Suetani ${ }^{4,1}$
}

\author{
Affiliations: \\ ${ }^{1}$ RIKEN CBS-TOYOTA Collaboration Center (BTCC), RIKEN Center for Brain Science, Wako, \\ Saitama, Japan. \\ ${ }^{2}$ Division of Neural Dynamics, Department of System Neuroscience, National Institute for \\ Physiological Sciences, National Institutes of Natural Sciences, 38 Nishigonaka, Myodaiji, \\ Okazaki, Aichi, 444-8585, Japan. \\ ${ }^{3}$ Department of Physiological Sciences, School of Life Science, The Graduate University for \\ Advanced Studies (SOKENDAI), 38 Nishigonaka, Myodaiji, Okazaki, 444-8585, Japan. \\ ${ }^{4}$ Faculty of Science and Technology, Oita University, 700 Dannoharu Oita, 870-1192, Japan. \\ *Correspondence to: Keiichi Kitajo, kkitajo@nips.ac.jp
}

\begin{abstract}
:
It is an open question as to whether macroscopic human brain responses to repeatedly presented external inputs show consistent patterns across trials. We here provide experimental evidence that human brain responses to noisy time-varying visual inputs, as measured by scalp electroencephalography (EEG), show a signature of consistency. The results indicate that the
\end{abstract}


EEG-recorded responses are robust against fluctuating ongoing activity, and that they respond to visual stimuli in a repeatable manner. This consistency presumably mediates robust information processing in the brain. Moreover, the EEG response waveforms were discriminable between individuals, and were invariant over a number of days within individuals. We reveal that time-varying noisy visual inputs can harness macroscopic brain dynamics and can manifest hidden individual variations.

One Sentence Summary: We provide experimental evidence that human brain responses to noisy visual inputs show trial-to-trial consistency in an individual way.

\section{Main Text:}

The brain is a complex system composed of a number of nonlinear elements (i.e., neurons) inter-connected with excitatory and inhibitory connections. From the viewpoint of computational neuroscience, it is known that model networks of such complex systems produce irregular and chaotic activity due to a balance of excitatory and inhibitory connections $(1,2)$. Prior experimental studies have reported fluctuating ongoing activity in the brain, even without specific external stimuli $(3,4)$. Theoretical studies on chaotic dynamics have revealed that small differences in the initial states of a nonlinear dynamical system can lead to large changes in the responses of the system, even when the system is driven by the same external inputs. It is therefore an intriguing question as to whether consistent neural responses to repeatedly presented identical external inputs are observable in brain dynamics with ongoing fluctuations. In this context, the reliability of spike timing, which is defined as the repeatability of the timing of 
neuronal spikes when a single neuron is repeatedly driven by a time-varying identical input, is of great interest to neuroscientists. Spike timing should provide "reliable" information in an information processing sense, especially if the spikes sequentially produce identical changes in postsynaptic neurons, and this repeatability of timing holds in whole neuronal circuits. Notably, a pioneering study using rat cortical slices showed that single-neuron spikes responding to a repeatedly injected noisy current input showed highly consistent patterns with high temporal precision across trials (5). In addition, an in vivo monkey study showed that individual neurons in middle temporal area responding to repeated presentations of the same noisy time-varying motion stimulus exhibited synchronized spike patterns across trials, with high timing precision (6). Moreover, a theoretical study showed that intermittent consistent responses in spike timing should be observable in neural networks with chaotic dynamics (2). Although there is ongoing debate on spike-timing reliability in relation to the rate coding idea (7), these prior studies indicate the existence of reliability in spike timing mediated by the consistent responses of dynamical systems to time-varying inputs.

From a nonlinear dynamical system theory viewpoint, one that is not necessarily concerned with neuronal spikes, this feature is called "consistency", and is defined as the inter-trial repeatability of response waveforms of a system that is being repeatedly driven by the same fluctuating input signal, which can include noise as well as chaotic signals. Consistency has been experimentally demonstrated in laser systems (8), and numerically in the chaotic Lorenz model (9). Consistency is counterintuitive, because it occurs when initial conditions differ, even in chaotic dynamical systems whose responses are sensitive to initial conditions. Although local field potential (LFP) recordings and electroencephalography (EEG) are spatially crude methods compared with spike recordings of single neurons, they reflect dynamic changes in the 
excitability of neuronal ensembles associated with synchronous spike inputs in neuronal circuits. Therefore, consistency in EEG waveforms could be associated with information processing mediated by a number of spike communications. However, to our knowledge, the presence of inter-trial consistency in macroscopic brain responses has not been extensively tested. It remains an open question as to whether the large-scale neural activity measured by EEG of the intact human brain shows a consistent nature. To this end, we investigated whether scalp EEG-assessed macroscopic human brain responses to an identical noisy visual input showed trial-to-trial consistency on an individual basis.

Participants $(n=130)$ were presented with a noisy flickering checkerboard stimulus and required to look passively at the stimulus. The gray-level contrast between adjacent squares was temporally modulated according to the Gaussian white noise with one of five different standard deviations (mean $=0, \mathrm{SD}=16,32,48,64,80$, in 8-bit gray level) and one of two noise realizations. Participants were repeatedly presented with each of ten presentations of noisy flickering for 14 times in a randomized order. Fig. 1 indicates the time course of the visual stimuli. The visual stimuli started with a fixation cross, which was followed by a $2.5 \mathrm{~s}$ static checkerboard stimulus, and then by a noisy flickering checkboard for $5.5 \mathrm{~s}$. Using a 63-channel EEG amplifier, we recorded high-density scalp EEG signals while participants either viewed the noisy checkerboard or rested for $3 \mathrm{~min}$.

To assess the degree of consistency of brain responses, we applied a canonical correlation analysis (CCA)-based method between the pairwise EEG epochs within and across individuals. CCA is a conventional statistical method for extracting the linear combinations of data variables that give maximal correlations between pairwise datasets (10), and is useful for detecting synchronization between time series data from two dynamical systems such as two coupled 
chaotic systems (11). We used CCA to extract correlated components between pairs of EEG trials, with these correlated components being considered to reflect consistency in the nature of the brain activity. Fig. 2 indicates the analytical pipeline for the CCA-based method. Specifically, we extracted canonical variates composed of linear combinations of EEG signals using the 1st eigenvector of the CCA as weight coefficients, which indicated the maximal correlations between two EEG epochs consisting of 63 channels and 5500 samples per channel. As there were 14 epochs for each of the two realizations of noise, we obtained a $28 \times 28$ L1 distance matrix for each of the 130 participants for each noise intensity level. Next, we applied classical multidimensional scaling (MDS) to analyze the Mahalanobis distance between the centroids of two classes (noise presentation 1 vs 2) and the classification accuracy using a linear support vector machine (SVM) (12) with leave-one-out cross validation (LOOCV) in two-dimensional MDS space.

Fig. 3 demonstrates representative 1st canonical variates extracted from two EEG epochs from a single participant for identical (Fig. 3A) and different flickering presentations (Fig. 3B). Although the CCA-based method will try to extract correlated variates from any dataset, the 1st canonical variates derived from a pair of EEG epochs corresponding to the same presentation of visual noise show a much higher canonical correlation than a pair of epochs corresponding to different presentations of visual noise. The 1st canonical variates extracted from the paired EEG epochs for the same visual noise presentations showed peaks in the 4-8 Hz theta band (Fig. S1). Fig. 3B indicates group data for canonical correlation coefficients between the paired EEG epochs for the same and different visual noise presentations averaged across all 130 participants. We observed that the noise level had significant effects (Friedman test, $F_{r}(5,645)=375.59, p$ $<0.001)$ on the canonical correlations. Post-hoc multiple comparison tests showed that the 
condition with noise of SD 80 showed higher canonical correlations than the other five lower noise levels for identical visual noise presentations (Wilcoxon signed-rank test, two-sided, Bonferroni corrected $p<0.001)$. Canonical correlations for the EEG trials for two distinct noise presentations also differed across noise levels (Friedman test, $F_{r}(5,645)=33.31, p<0.001$; Fig. 4B). In addition, the canonical correlations from EEG trials with the same noise inputs were statistically higher than those for different noise inputs for the five noise levels (noise SD: 16, 32, 48, 64, 80) (Wilcoxon signed-rank test, two-sided, Bonferroni corrected $p<0.001$ ). Next, to investigate topographical patterns in the extent of contributions of EEG signals to the canonical variates, we assessed the canonical loading, which is the correlation between projected canonical variates and EEG signals. Fig. 3 D shows the topography of absolute values of canonical loading averaged across all participants for noise with a SD of 80 . We found prominent signal contributions from occipital electrodes placed over the lower visual cortex. These results suggest that this consistency phenomenon mainly occurs in the lower visual areas, and that the CCA-based method did not extract merely spurious correlations between the paired EEG epochs for the same noise presentations by overfitting the EEG signals from all electrodes.

Next, we analyzed trial-to-trial distance matrices estimated as the L1 norm of two canonical variates and analyzed how EEG trials for distinct noise presentations were located in classical MDS space. Fig. 4A shows a MDS visualization of EEG responses from a representative participant for two noise presentations at different noise levels. We found a clear separation between EEG epochs from two different visual noise inputs in high noise intensity conditions. To assess the similarity of EEG responses to identical visual inputs in comparison with those to different visual inputs, we estimated the Mahalanobis distance between the centroids of EEG trials for two distinct noise presentations in all participants, with this being the 
ratio of within-label (noise) variance to across-label (noise) variance in the two-dimensional MDS space. Fig. 4B shows the Mahalanobis distances for EEG trials for two distinct visual noise conditions at all noise levels averaged across all participants. The Mahalanobis distance monotonically increased as a function of noise intensity, and we observed significant effects of noise intensity on the Mahalanobis distance (Friedman test, $\left.F_{r}(5,645)=422.5 p<0.001\right)$. Post-hoc multiple comparison tests showed that noise with a SD of 80 showed higher Mahalanobis distances between two noise presentations than did the other noise levels (Fig. 4B; Wilcoxon signed-rank test, two-sided, Bonferroni corrected, $p<0.001)$. We also used a linear SVM with LOOCV in the classical MDS space to analyze the classification performance of EEG trials corresponding to two visual stimuli. The classification accuracy increased as a function of noise, up to a median correct rate of $100 \%$ for noise of SD 48, 64,80 , and we found that the noise intensity had significant effects on the LOOCV accuracy (Friedman test, $F_{r}(5,645)=$ 305.1812, $p=0.001$ ) (Fig. 4B). Post-hoc multiple comparison tests showed that noise with a SD of 80 showed higher LOOCV accuracy than all other noise levels (Wilcoxon signed-rank test, two-sided, Bonferroni corrected $p<0.001$; Fig. 4C). These results indicate that noise with higher intensity showed clear separation of EEG trials corresponding to two different noise presentations, and high classification performance on a single-trial basis.

Taken together, the intra-individual analyses indicate that EEG responses to identical noisy visual inputs show trial-to-trial consistency with the response waveforms differing depending on the presentation of input signals. It should be noted that higher noise levels induced stronger consistency features. To our knowledge, this is the first evidence that human EEG-level neural signals show a consistency signature in response to noisy visual inputs, other than that from our earlier preliminary results on a small dataset (three participants) (13). 
Another intriguing question is whether individual brains, which should differ in various aspects such as network architecture and dynamical characteristics of neurons, show distinct EEG responses to identical noise inputs. To this end, we analyzed individual differences in EEG responses by conducting inter-individual CCA for identical visual inputs (Fig. 5A). Fig. 5B shows a MDS visualization of EEG responses for a pair of participants at different noise levels. The inter-individual differences revealed by separation of the individuals were most prominent for the highest noise level. Using the Mahalanobis distance, we estimated the separation of pairwise individuals in two-dimensional MDS space. We found that the noise level had significant effects on the Mahalanobis distance between the centroids of EEG trials for different individuals (Friedman test, $\left.F_{r}(5,41920)=33222, p<0.001\right)$. Post-hoc multiple comparison tests revealed that the Mahalanobis distance between participants was largest for the highest noise intensity (Fig. 5C; Wilcoxon signed-rank test, two-sided, Bonferroni corrected $p<0.001$ ). We also tested if a SVM could classify single-trial EEGs from different individuals using the LOOCV method in a verification context, and observed significant effects of noise level on the classification accuracy (Friedman test, $\left.F_{r}(5,41920)=26690, p<0.001\right)$. The SVM classifier showed a median LOOCV accuracy of $100 \%$ at the higher noise levels (SD 32, 48, 64, 80), with the highest noise (SD 80) showing higher LOOCV accuracy than all other lower noise conditions (Wilcoxon signed-rank test, two-sided Bonferroni corrected, $p<0.001$, Fig. 5D). These results indicate that the consistency nature was common across individuals, although the response waveforms differed across them.

Moreover, in 20 additional participants, the degree of inter-individual separation was higher in the case of noisy visual inputs than it was with periodic stimuli $(3.75 \mathrm{~Hz}, 5.0 \mathrm{~Hz}, 7.5$ 
$\mathrm{Hz}, 15.0 \mathrm{~Hz}$ ), as revealed by a significantly higher Mahalanobis distance between individuals and a higher SVM classification accuracy (Fig. S2).

We conducted a follow-up testing session in 32 participants at a mean interval of 101 days and using the individual-wise Mahalanobis distance, tested whether the 2nd recordings of follow-up showed a shorter Mahalanobis distance to the EEG responses of their 1st recordings than to those of other individuals by looking at the distance ranking of their 1 st recording data. Critically, top 1-ranked (shortest distance) participants were 28 out of 32 participants, and top 5-ranked participants were 32 participants among 32 participants (100\%) in the individual-wise identification. Fig. 6 shows a t-distributed stochastic neighbor embedding (t-SNE) (14) visualization of the EEG responses of participants. The normalized Mahalanobis distance to other individuals was used as the individual feature for the t-SNE. It can be observed that most of the 2 nd recordings of the 32 follow-up participants are located close to their 1 st recordings. These results indicate that within-individual noise-induced EEG responses are invariant across days and show high accuracy in the identification of individuals.

Taken together, the results of this study show that large-scale human neural activity exhibits a consistency nature in response to repeatedly presented noisy visual inputs. Scalp EEG is the filtered summation of postsynaptic membrane potentials from a large number of cortical neurons, and although it is a crude method in terms of its spatial resolution, a number of studies have shown that a variety of EEG dynamics, such as oscillations and synchrony, are important in mediating perceptual and cognitive processes associated with the spike communications underlying these processes. For example, Fries (15) proposed that coherence in membrane potentials between neuronal groups could be a fundamental basis for spike communication across neuronal groups. We therefore speculate that consistency in the EEG responses to time-varying 
inputs could be a fundamental basis for robust information processing in macroscopic neural activity, which can constrain spike communication. In fact, the response waveforms were input-dependent, as demonstrated by the clear separation between EEG responses corresponding to two distinct noise presentations.

Animal studies have shown that ongoing cortical activity exhibits a rich and high spatiotemporal variability, even under anesthesia, and that it modulates evoked responses (3). Ongoing activity in the orientation columns of cat visual cortex exhibits instantaneous patterns that resemble stimulus-evoked orientation maps under anesthesia (4). Non-additive interaction between task-induced and ongoing BOLD activity has also been reported in humans $(16,17)$, and moment-to-moment variability in BOLD activity is associated with functional performance (18). Additionally, there have been human studies showing that perceptual performance is affected by moment-to-moment fluctuations in ongoing activity, such as the phase of ongoing EEG oscillations $(19,20)$. These previous studies suggest that ongoing activity could modulate responses to external stimuli and perceptual performance. However, differences in initial brain states caused by moment-to-moment fluctuations in ongoing activity could be a potential risk for stable neural responses if the fluctuations were uncontrolled and unrelated to the signals coding information. We speculate that the consistency mechanism observed in the current study reconciles the fluctuating nature of ongoing brain activity with robust information processing. In other words, EEG-level consistency could reflect robust macroscopic neural responses for perceptual information processing regardless of fluctuations in ongoing activity.

We also demonstrated the noise-induced EEG response waveforms were discriminable between individuals. We speculate that inter-individual differences in the human brain networks are associated with distinct temporal patterns related to identical presentations of noisy inputs 
across individuals. It should also be noted that the individual dynamical features could still be detected an average of 3 months later, suggesting that they are robust and associated with individuality. It is also intriguing that the neural code for time-varying visual signals triggered by such a simple checkerboard structure showed individual differences.

From the viewpoint of nonlinear science, a variety of nontrivial noise-induced dynamics have been studied, such as stochastic resonance $(21,22)$, noise-induced order (23), consistency $(8,9)$, and noise-induced synchronization in chaotic oscillators (24), excitable media (25), and periodic phase oscillators (26). In the field of computational neuroscience, it is an open question as to whether these noise-induced nonlinear phenomena play computational roles mediating information processing in the brain. For example, stochastic resonance (SR), which is a counterintuitive phenomenon where an optimal level of noise can enhance the responses of a nonlinear system to weak inputs, has been shown to play functional roles in animal and human brains. SR has been observed to improve sensitivity to sensory inputs in crayfish mechanoreceptor cells (27), paddle fish feeding behavior (28), and human visuo-motor coupling $(29,30)$. Apart from SR, there is only sparse evidence of other noise-induced phenomena associated with information processing in the brain, besides the above-mentioned single cell-level reliability studies $(5,6)$. We hereby add another piece of evidence that noise-induced dynamics in macroscopic brain responses are observable and robust, and that they can play roles in information processing in the brain.

One of the efficient ways to probe the internal state or structure of such a complex system is to "ping" the system with a pulse input. Such perturbation approaches have been widely undertaken in physical, chemical, and biological systems, including the human brain. For example, transcranial magnetic stimulation (TMS) with concurrent EEG recording is used to 
probe human brain features such as effective connectivity (31) and area-dependent natural frequencies (32). We also reported a repetitive TMS paradigm to probe phase-amplitude coupling with concurrent EEG recording (33). In addition, Wolff et al. recently reported a new impulse response paradigm to probe the instantaneous hidden neural state associated with working memory (34). The frequency tagging method has also been of wide use for probing processing-related areas in the visual system $(35,36)$. These studies indicate that perturbation approaches provide versatile tools for understanding the dynamical features of the brain. However, as far as we know, no prior studies have used noisy continuous inputs to probe the dynamical nature of the human brain. Thus, this study is novel in that it provides empirical evidence that noisy inputs can probe the dynamics of the brain, irrespective of differences in its initial condition, in a system- and input-dependent way.

Recent human functional magnetic resonance imaging (fMRI) studies show that functional connectivity profiles during rest or task performance work well as a "fingerprint" to identify individuals (37). Compared with fMRI, EEG is a crude method in terms of spatial resolution. It is therefore surprising that, despite the potential misalignment of electrode locations and different physiological conditions over sessions, the results were stable over periods of days. Such stability is sometimes an issue in practical brain machine interfaces (38). We speculate that our dynamical method takes advantage of EEG-level neural dynamics, which show higher temporal precision than fMRI, despite the low spatial resolution. Recently, there was an EEG fingerprint study utilizing event-related potentials (ERPs) as individual signatures (39).

ERP-based methods, however, need a number of trials (50-100 times) to allow a clear ERP to be attained. By contrast, in our novel method, a $5.5 \mathrm{~s}$ single trial is enough to obtain sufficient performance in individual verification if there is a target "database" to check. This advantage of 
our method over ERP-based methods could facilitate versatile applications in brain machine interface field, because this field often relies on the consistency of neural responses.

\section{References and Notes:}

1. C. van Vreeswijk, H. Sompolinsky, Neural Comput. 10, 1321(1998).

2. G. Lajoie, K. K. Lin, E. Shea-Brown. Phys Rev E Stat Nonlin Soft Matter Phys. 87, 052901 (2013).

3. A. Arieli, A. Sterkin, A. Grinvald, A. Aertsen. Science 273, 1868-1871 (1996).

4. T. Kenet, D. Bibitchkov, M. Tsodyks, A. Grinvald, A. Arieli, Nature 425, 954-956 (2003).

5. Z. F. Mainen, T. J. Sejnowski, Science 268, 1503-1506 (1995).

6. G. T. Buraĉas, A. M. Zador, M. R. DeWeese, T. D. Albright, Neuron 20, 959-969 (1998).

7. M. London, A. Roth, L. Beeren, M. Hausser, P. E. Latham, Nature 466, 123-127 (2010).

8. A. Uchida, R. McAllister, R. Roy, Phys. Rev. Lett. 93, 244102 (2004).

9. A. Uchida, K. Yoshimura, P. Davis, S. Yoshimori, R. Roy, Phys Rev E Stat Nonlin Soft Matter Phys. 78, 036203 (2008).

10. H. Hotelling, Biometrika 28, 321-377 (1936).

11. H. Suetani, Y. Iba, K. Aihara, Journal of Physics A: Mathematical and General 39, 10723-10742 (2006).

12. C. Cortes, V. Vapnik, Machine Learning 20, 273 (1995). 
13. K. Kitajo, H. Suetani, Proceedings of International Symposium on Nonlinear Theory and Its Applications (NOLTA) 2014, 443-445 (2014).

14. L. J. P. v. d. Maaten, G. E. Hinton, Journal of Machine Learning Research 9, 2579-2605 (2008).

15. P. Fries, Trends in cognitive sciences 9, 474-480 (2005).

16. B. J. He, J. Neurosci. 33, 4672-4682 (2013).

17. G. Northoff, P. Qin, T. Nakao, Trends Neurosci. 33, 277-284 (2010).

18. D. D. Garrett et al., Neuroscience and biobehavioral reviews 37, 610-624 (2013).

19. N. A. Busch, J. Dubois, R. VanRullen, J. Neurosci. 29, 7869-7876 (2009).

20. K. E. Mathewson, G. Gratton, M. Fabiani, D. M. Beck, T. Ro, J. Neurosci. 29, 2725-2732 (2009).

21. K. Wiesenfeld, F. Moss, Nature 373, 33-36 (1995).

22. L. Gammaitoni, P. Hänggi, P. Jung, F. Marchesoni, Reviews of Modern Physics 70, 223-287 (1998).

23. K. Matsumoto, I. Tsuda, Journal of Statistical Physics 31, 87-106 (1983).

24. A.S. Pikovsky, in Nonlinear and Turbulent Processes in Physics, R. Z. Sagdeev Ed. (Harwood Acad., Chur, Switzerland, 1984),

25. A. Neiman, L. Schimansky-Geier, A. Cornell-Bell, F. Moss, Physical Review Letters 83, 4896-4899 (1999).

26. J. N. Teramae, D. Tanaka, Phys. Rev. Lett. 93, 204103 (2004).

27. J. K. Douglass, L. Wilkens, E. Pantazelou, F. Moss, Nature 365, 337-340 (1993). 
28. D. F. Russell, L. A. Wilkens, F. Moss, Nature 402, 291-294 (1999).

29. K. Kitajo, D. Nozaki, L. M. Ward, Y. Yamamoto, Phys. Rev. Lett. 90, 218103 (2003).

30. K. Kitajo et al., Europhysics Letters (EPL) 80, 40009 (2007).

31. M. Massimini et al., Science 309, 2228-2232 (2005).

32. M. Rosanova et al., J. Neurosci. 29, 7679-7685 (2009).

33. S. Glim et al., Neural Plast. 2019, 1-13 (2019)

34. M. J. Wolff, J. Jochim, E. G. Akyurek, M. G. Stokes, Nat. Neurosci. 20, 864-871 (2017).

35. G. Tononi et al., Proc. Natl. Acad. Sci. U. S. A. 95, 3198-3203 (1998).

36. A. M. Norcia, L. G. Appelbaum, J. M. Ales, B. R. Cottereau, B. Rossion, J Vision 15, 4 (2015).

37. E. S. Finn et al., Nat. Neurosci. 18, 1664-1671 (2015).

38. H. Morioka et al., Neuroimage 111, 167-178 (2015).

39. B. C. Armstrong et al., Neurocomputing 166, 59-67 (2015).

Acknowledgments: We thank Mr. Motonobu Fujioka and Mr. Shunta Yoshimura for help with the data analysis. We thank Dr. Mitsuo Kawato for helpful discussion and comments.

Funding: This study was partially supported by a research grant from the ImPACT Program of the Council for Science, Technology and Innovation (Cabinet Office, Government of Japan), and JSPS Grand-in-Aid for Scientific Research (B) (23300218).

Author contributions: KK contributed to conceptualization, formal analysis, funding acquisition, investigation, methodology, project administration, resources, software, supervision, validation, 
visualization, writing - original draft, writing - review and editing. TS contributed to formal analysis, software. YM contributed to investigation. HS contributed to conceptualization, formal analysis, funding acquisition, investigation, methodology, software, validation, visualization, writing - review and editing.

Competing interests: KK and HS hold the patent pending (PCT/ US15/576632 JP2017-520817) entitled "determination device, determination method, program, and information storage medium".

Data and materials availability: All data, code, and materials used in the analysis are available upon request.

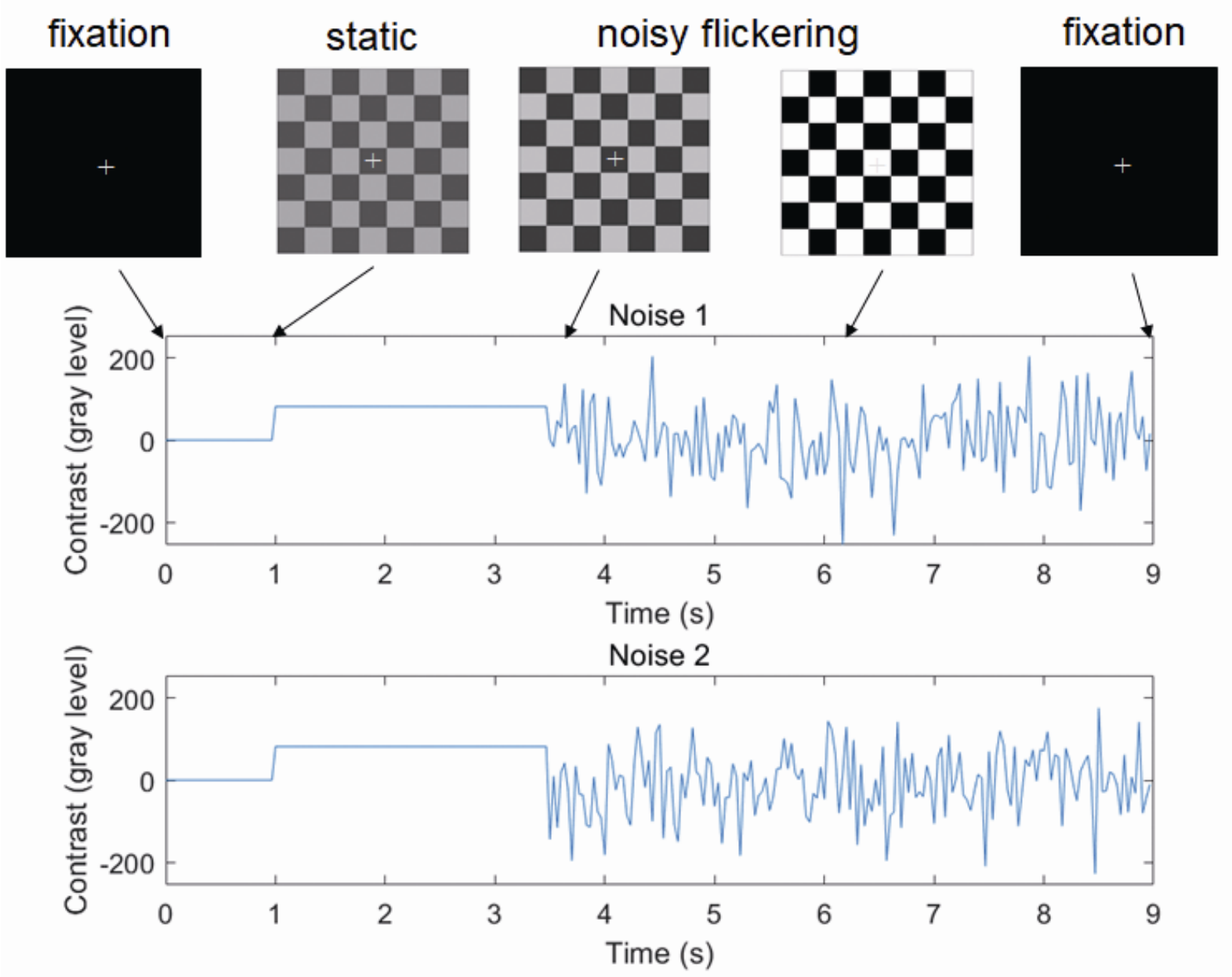


Figure 1. A schematic presentation of visual stimuli and experimental paradigm

The stimuli consisted of a fixation cross, a static checkerboard (2.5 s), and a noisy flickering checkerboard (5.5 s). 


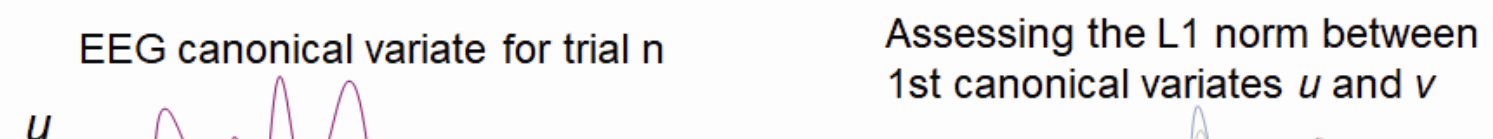

EEG trial-wise distance matrix

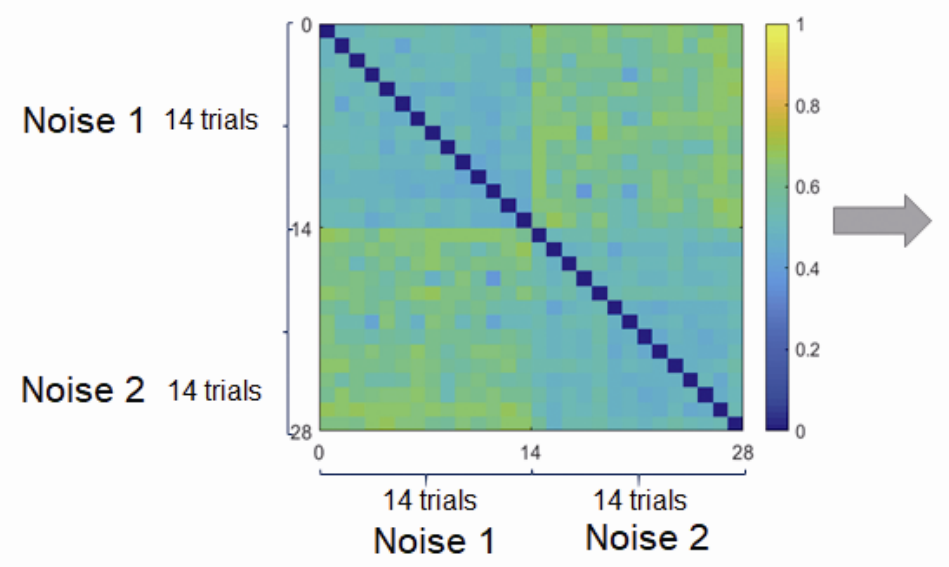

1 st canonical variates $u$ and $v$
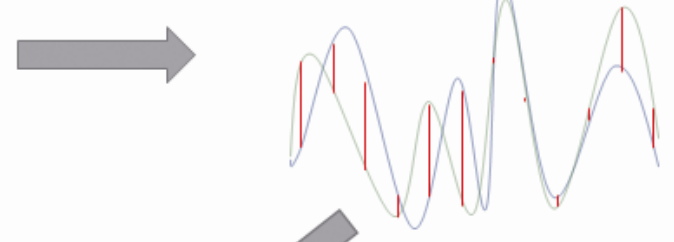

Classical MDS

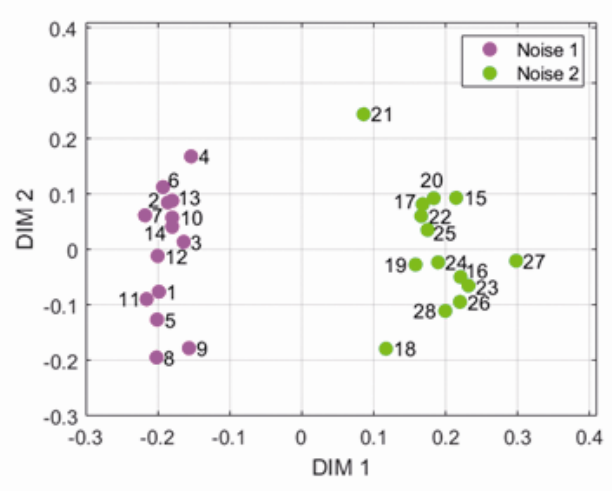

Figure 2. Schematic presentation of the analytical pipeline for the intra-individual analysis

Classical multidimensional scaling (MDS) was conducted using a distance matrix composed of $28 \times 28$ L1 norm values between EEG canonical variates, and how EEG trials were located in two-dimensional space was visualized. 
A Same noise presentations

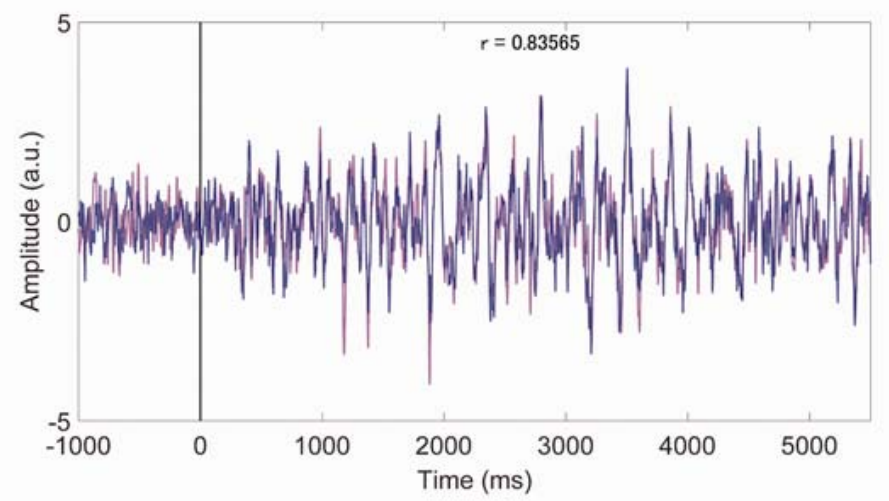

B Different noise presentations

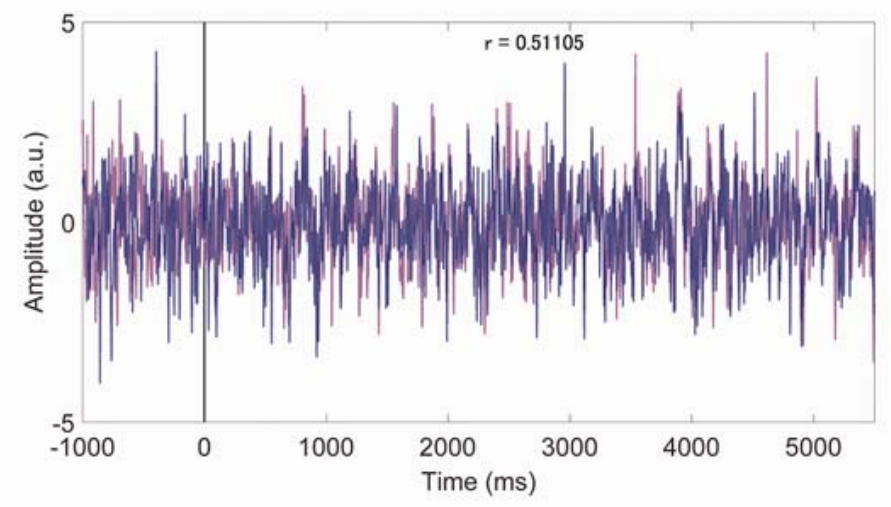

C

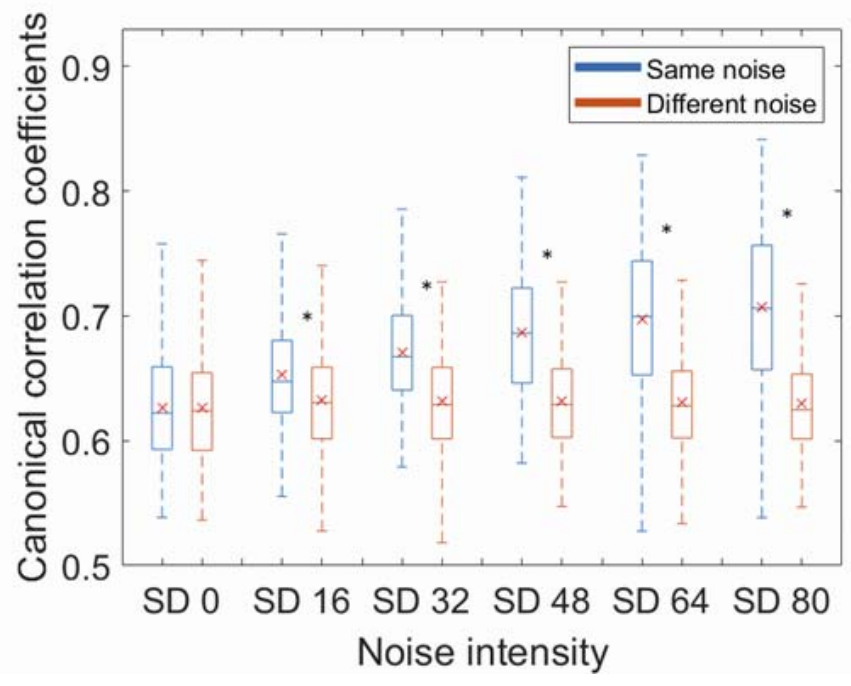

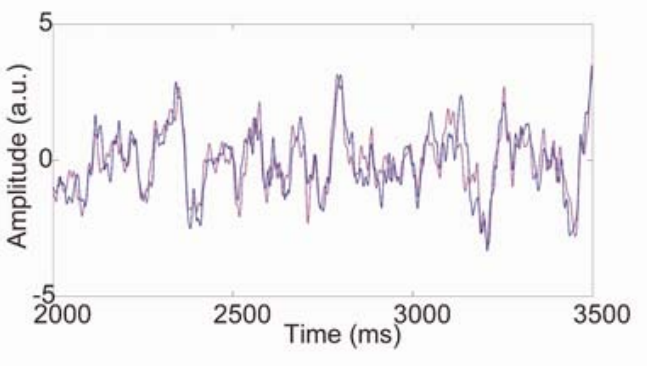
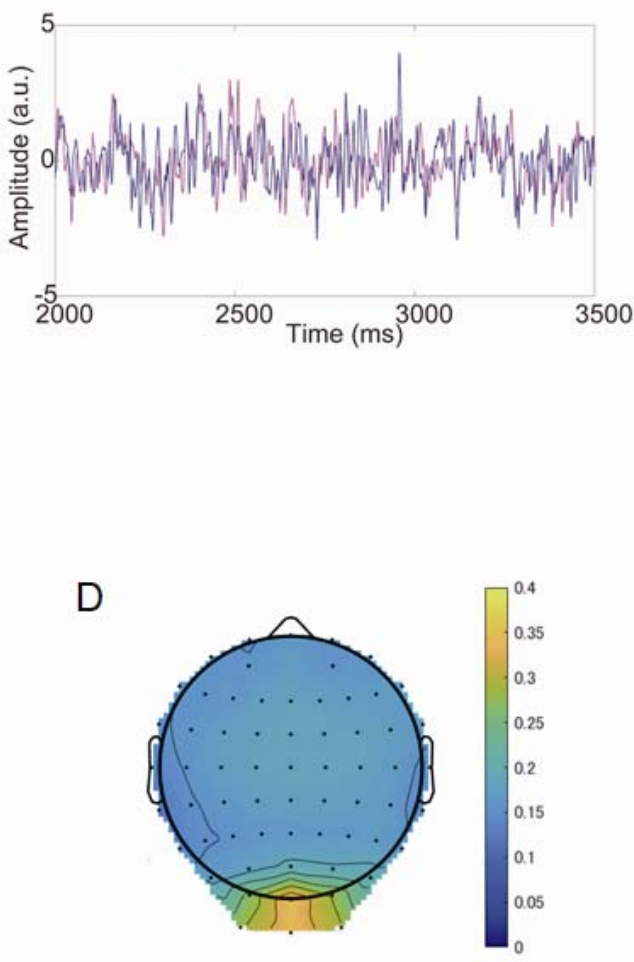

Figure 3. Representative data of EEG canonical variates

Representative 1st canonical variates from an individual for two distinct pairs of EEG trials. 
(A) A pair of EEG canonical variates extracted from a pair of trials where the participant viewed the same presentation of visual noise.

(B) Another pair of EEG canonical variates extracted from trials with different presentations of noise. Visual flicker starts at $0 \mathrm{~ms}$. Right panels are magnified views of the corresponding EEG canonical variates from 2000 to $3500 \mathrm{~ms}$.

(C) Group data for canonical correlation coefficients for EEG trials for the same and different noise presentations as a function of noise intensity. Boxplots show the median, 25 and $75 \%$ quartiles (boxes), 1.5 times the interquartile range (whiskers), and mean (x). Asterisks indicate significant differences between same and different noise presentations.

(D) The topography of absolute values of canonical loadings averaged across all participants ( $n$ $=130$ ) for the noise SD 80 condition. Higher loadings were observed for occipital electrodes over the lower visual area. 
A Noise SD 0

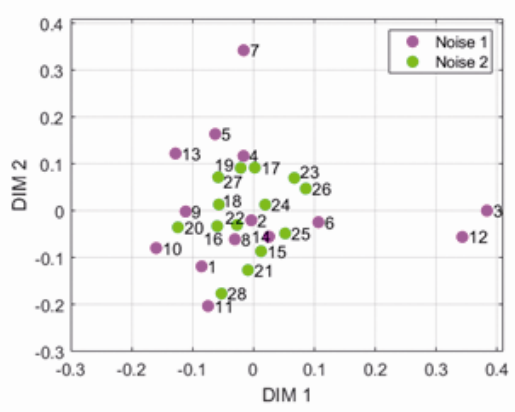

Noise SD 48

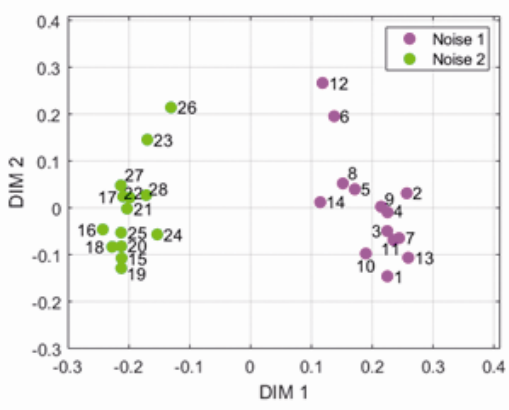

B

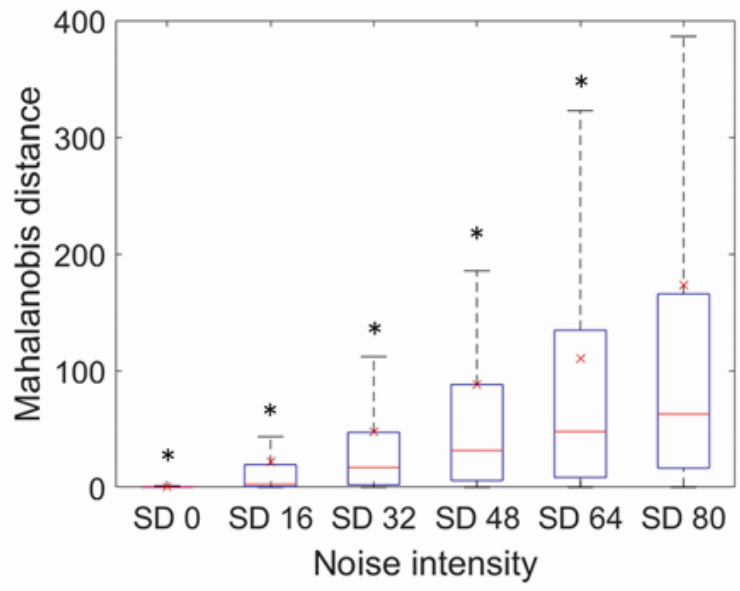

Noise SD 16

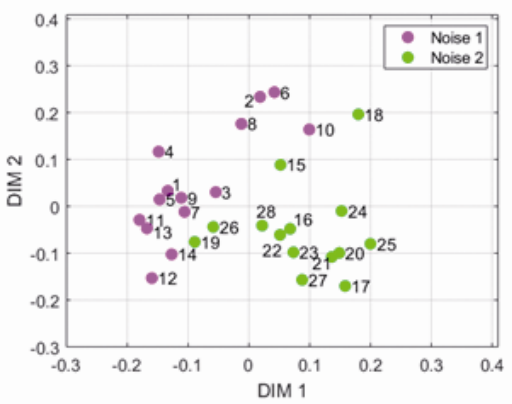

Noise SD 64

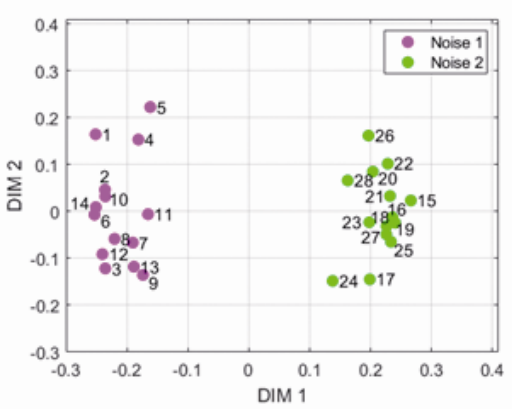

Noise SD 32

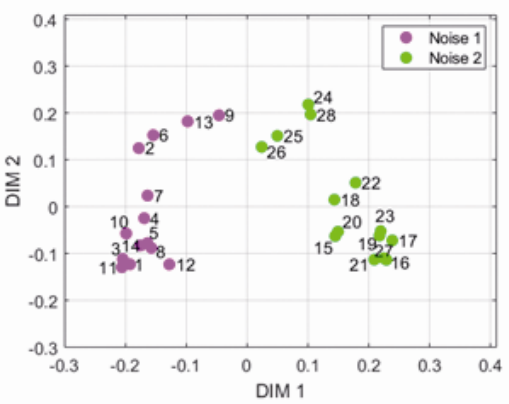

Noise SD 80

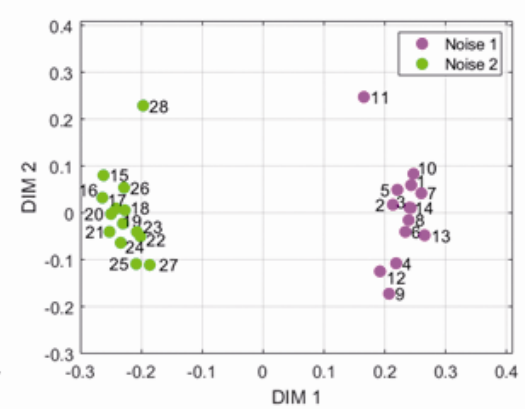

C

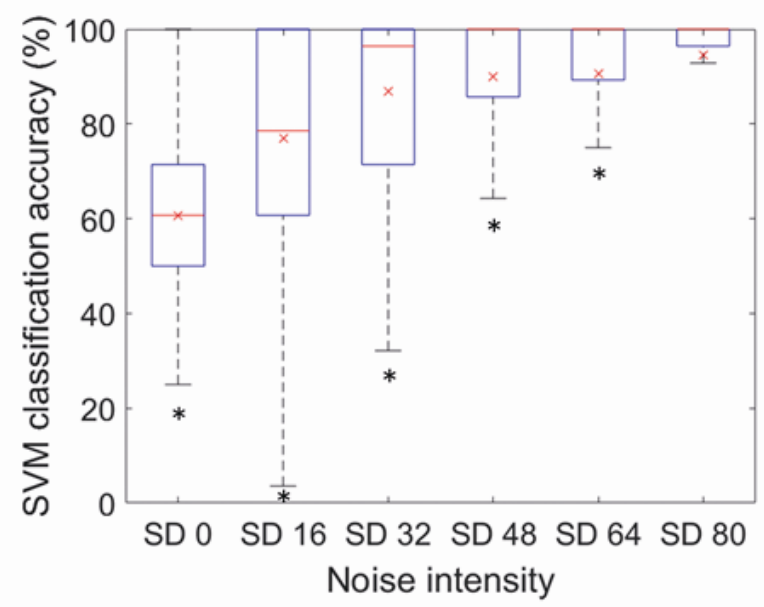

Figure 4. Intra-individual separation of EEG responses corresponding to two noise presentations

(A) Effects of noise intensity on separation of EEG trials for two distinct noise presentations.

Data from a representative participant. Markers in different colors correspond to different presentations of noise. The numbers indicate the trial number for noise presentations 1 (1-14) and 2 (15-28). 
(B) Group data for Mahalanobis distance between EEG responses for two noise presentations as a function of noise intensity $(n=130)$.

(C) Group data for SVM classification accuracy in MDS space as a function of noise intensity ( $n$ $=130)$.

Box plots in (B), (C) show the median, 25 and $75 \%$ quartiles (boxes), 1.5 times the interquartile range (whiskers), and mean (x). Asterisks indicate significant differences between SD 80 and other conditions. 
A

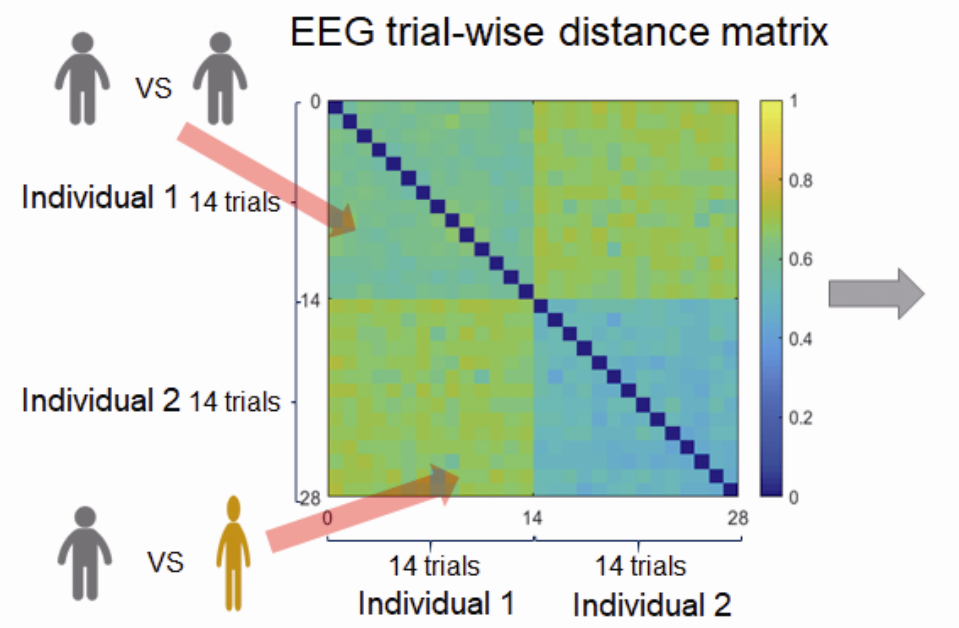

Classical MDS

B

Noise SD 0

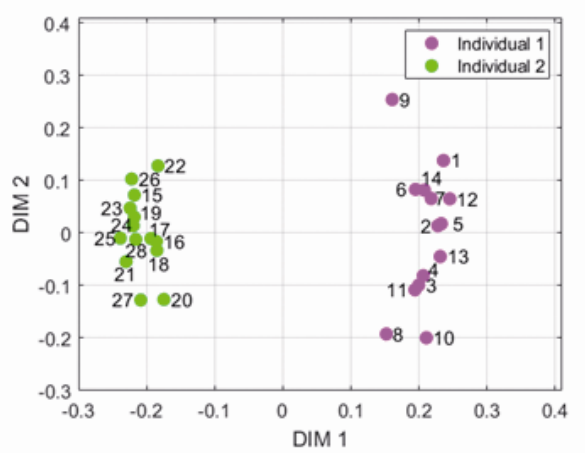

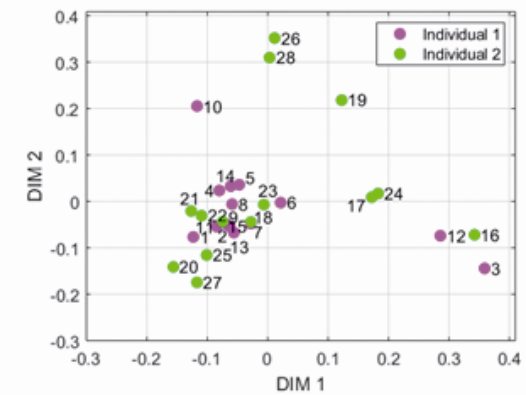

Noise SD 48
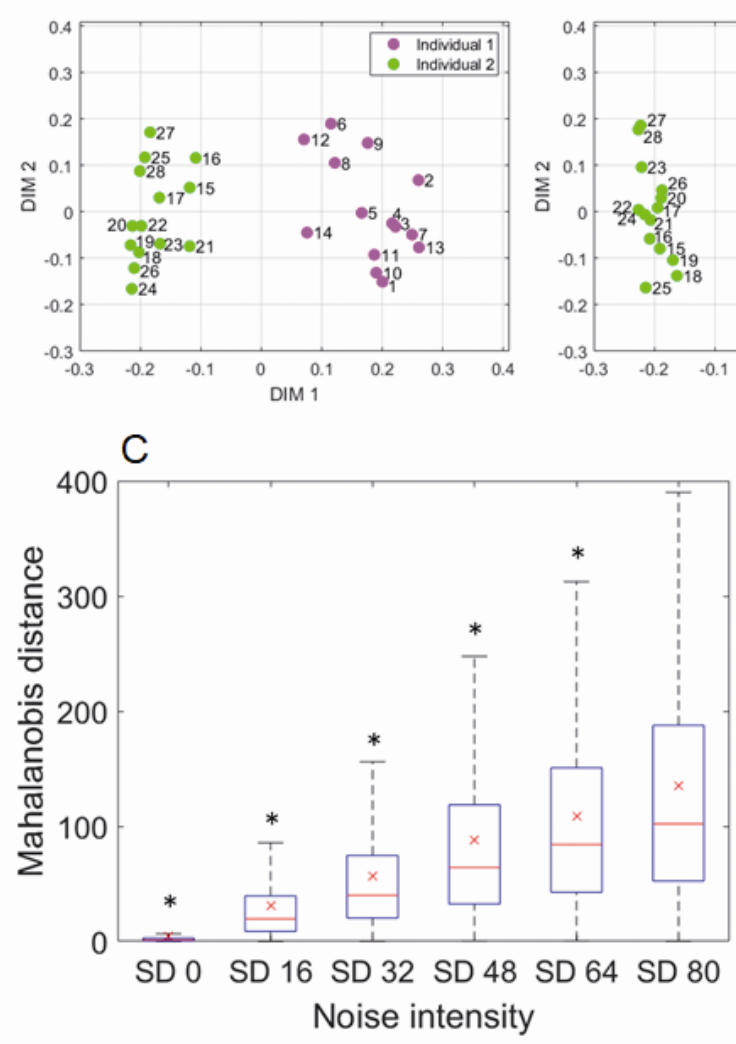

Noise SD 16

Noise SD 32

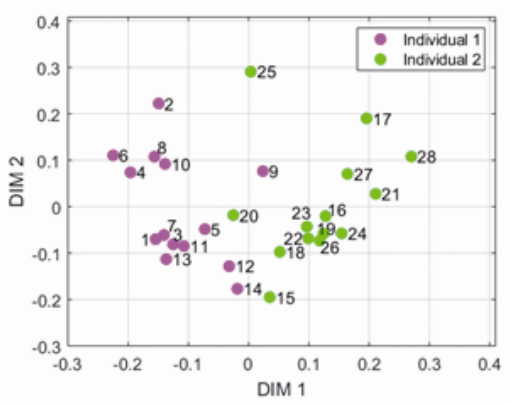

Noise SD 64
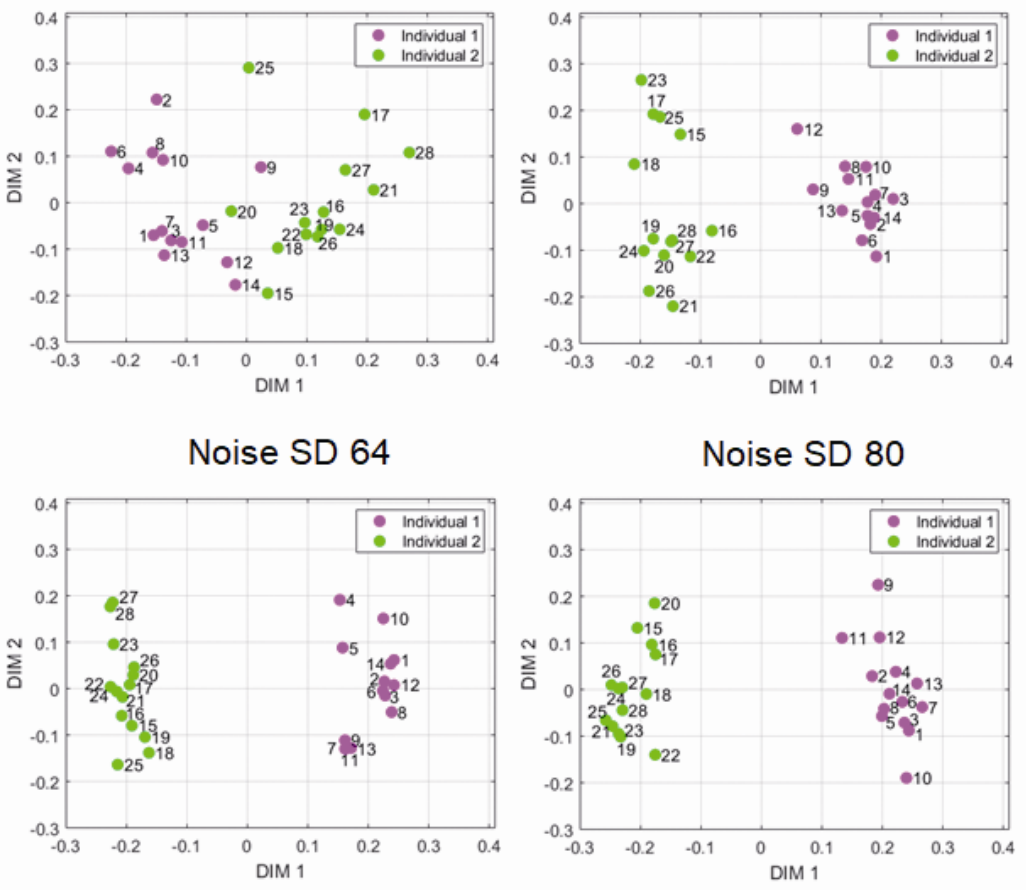

Noise SD 80
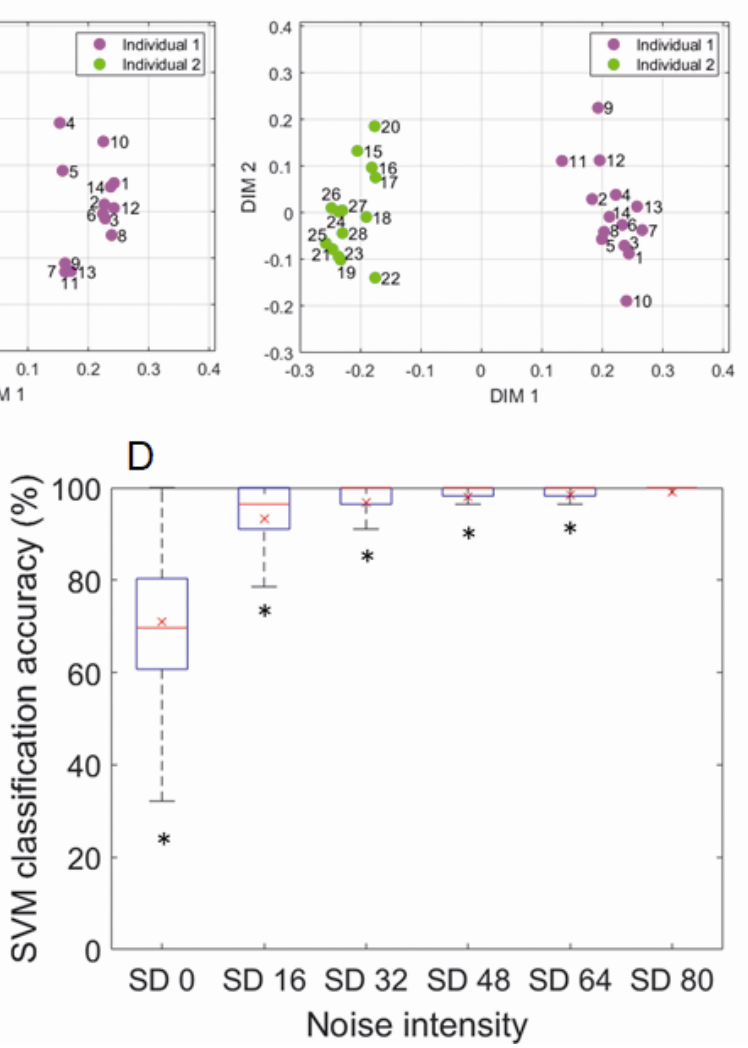
Figure 5. Inter-individual separation of EEG responses

(A) Schematic presentation of the distance matrix and MDS visualization.

(B) Effects of noise intensity on separation of EEG trials for two distinct individuals for presentations of the same noise. Data are from a pair of representative participants for distinct noise levels.

(C) Group data for the Mahalanobis distance obtained from pairs of the 130 participants for different visual noise intensities $\left(n=8385\left(={ }_{130} C_{2}\right)\right.$ participant pairs $)$.

(D) Group data for SVM classification accuracy in MDS space as a function of noise intensity $(n$ $=8385\left(={ }_{130} \mathrm{C}_{2}\right)$ participant pairs $)$. Box plots in $(\mathrm{C}),(\mathrm{D})$ show median, 25 and $75 \%$ quartiles (boxes), range (whiskers), and mean (x). Asterisks indicate significant differences between SD 80 and other conditions. 


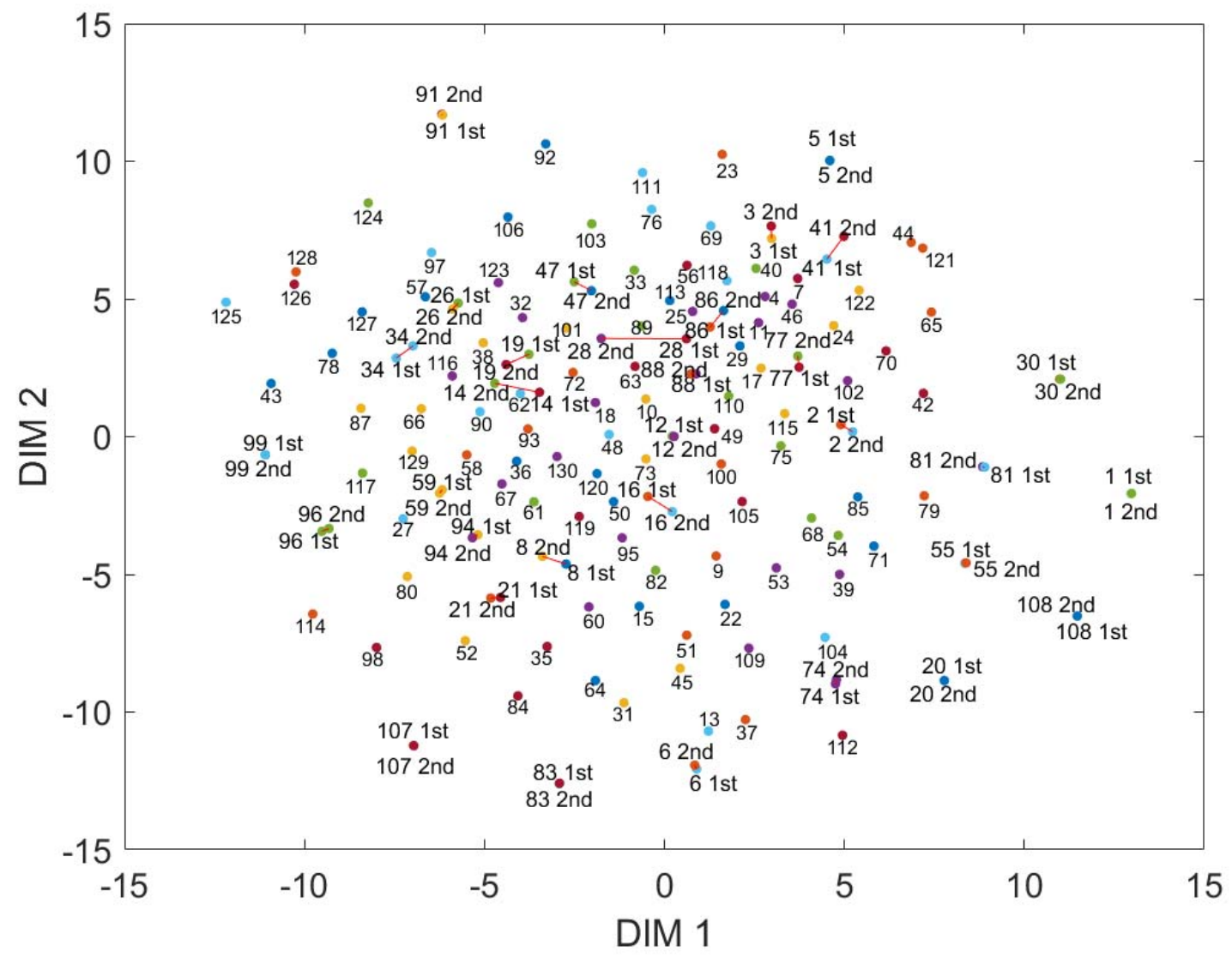

Figure 6. t-distributed stochastic neighbor embedding (t-SNE) visualization of the 130 initial individuals and 32 follow-up individuals

Individuals who underwent follow-up sessions are denoted using 1st and 2nd, in addition to the individual number (1-130), and connections between 1st and 2nd sessions are made with red lines, although most of the follow-up points overlap in t-SNE space (perplexity: 30, exaggeration: 5). 